\title{
Spreading Dystonia in Probable Creutzfeldt- Jakob Disease with Serial Diffusion-Weighted Magnetic Resonance Images
}

\author{
Seung-Hwan Lee ${ }^{a}$ Sang-Il Suh ${ }^{b}$ Seong-Beom Koh ${ }^{c}$ \\ ${ }^{a}$ Department of Neurology, Kangwon National University College of Medicine, and Departments of \\ ${ }^{b}$ Diagnostic Radiology and ${ }^{\mathrm{C}}$ Neurology, Korea University College of Medicine, Seoul, Korea
}

\section{Dear Sir,}

Creutzfeld-Jakob disease (CJD) is a progressive spongiform encephalopathy that is mainly characterized by rapidly progressive dementia, myoclonus, ataxia, epilepsia partialis continua, visual disturbances and movement disorders [1-7]. Several movement disorders including myoclonus, dystonia, choreoathetosis, tremor and parkinsonism have been described in a significant number of patients with CJD [8]. However, dystonia as an early symptom is rare. We report a patient who showed generalized dystonia followed by focal dystonia in early-stage disease with concomitant lesions on diffusionweighted MR images (DWI).

\section{Case Report}

A 64-year-old woman admitted to the hospital with a 4-week history of behavior change, emotional instability, gait disturbance and twisting of the left upper extremity. She had no history of receiving a blood transfusion or growth hormones. The patient had no history of neurosurgical or ophthalmologic surgery and no family history of neurological disease. During the initial neurological examination on admission, the patient was lethargic and showed only a withdrawal response to painful stimulation; she had synchronous contractions of the left finger, wrist and elbow muscles, which caused a dystonic pos-
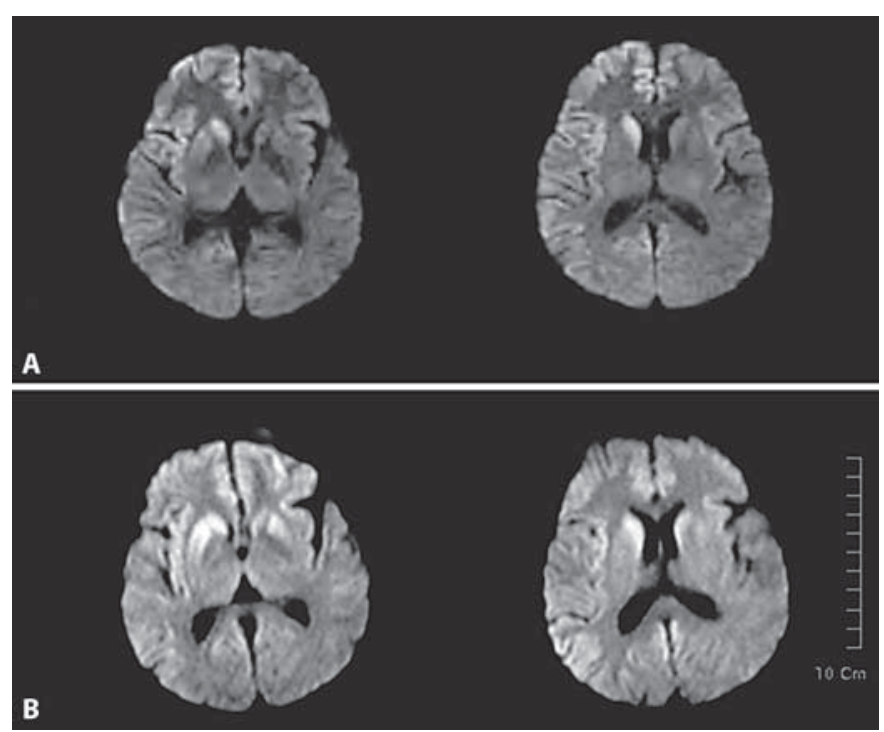

Fig. 1. DWI study. A The initial DWI shows increased signal intensities in the right striatum and in multiple cortical areas. B Ten-day follow-up DWI studies show increased signal intensity in both striatum and cortical areas.

turing of the left upper extremity. The dystonic posture of the left upper extremity caused levitation of the arm with flexed fingers and an extended wrist. Stimulussensitive generalized myoclonic jerks were also observed. The initial DWI scans on admission showed high signal intensity at the right caudate nucleus, putamen and frontal cortex (fig. 1A). Laboratory testing included a complete blood count, blood

\section{KARGER}

Fax +41613061234 E-Mail karger@karger.ch www.karger.com
(C) 2007 S. Karger AG, Base 0014-3022/07/0582-0122\$23.50/0 www.karger.com/ene
Seong-Beom Koh, MD, PhD

Department of Neurology, Korea University Guro Hospital

Korea University College of Medicine, 80 Guro-dong, Guro-gu

Seoul 152-703 (Korea)

Tel. +82 2818 6706, Fax +82 2818 6943, E-Mail parkinson@korea.ac.kr 
chemistry, thyroid function test, venereal disease research laboratory test, vasculitis markers, HIV antibodies, vitamin $\mathrm{B}_{12}$ and folate level; all were within normal limits. A spinal tap was performed; the cerebrospinal fluid had a normal opening pressure with a leukocyte count of $0 / \mathrm{mm}^{3}$, a protein level of $46 \mathrm{mg} / \mathrm{dl}$ and a glucose level of $106 \mathrm{mg} / \mathrm{dl}$. However, the Western blot test for the 14-3-3 protein, in the cerebrospinal fluid, was positive. An initial electroencephalogram (EEG) demonstrated a diffuse slow basal rhythm and periodic asymmetrical left hemispheric sharp waves (fig. 2A). After 2 weeks, the left periodic hemispheric sharp waves evolved to periodic bilateral synchronous sharp waves (fig. 2B).

The dystonic posture and myoclonic jerks worsened even with anticonvulsive therapy (valproic acid $600 \mathrm{mg} /$ day, clonazepam $0.5 \mathrm{mg}$ /day). Ten days after admission to the hospital, the dystonic posture spread over the right side to include the lower extremity. The follow-up DWI scans showed high signal intensity bilaterally at the basal ganglia and cortex (fig. 1B). Five weeks after admission, the patient became comatose; the myoclonic jerks and dystonia disappeared. She died 3 months later.

\section{Discussion}

CJD belongs to a group of disorders referred to as transmissible spongiform encephalopathy. The clinical manifestations of CJD vary. Early-stage disease is characterized by behavioral change, deterioration in intellectual function and cerebellar dysfunction [9]. Approximately 90\% of patients with CJD present with movement disorders; generalized myoclonus is common in advanced-stage disease [8]. However, other focal involuntary movements are rare, especially in early-stage disease. Dystonia as an early symptom of CJD is quite rare as well, but when present, it is usually unilateral with distal distribution. Several CJD cases presenting with focal dystonia have been reported [2, 3, 6]. In these reported cases, dystonia was associated with other movement disorders such as focal myoclonus, focal or generalized chorea, tremor and bradykinesia. Dystonia progressively evolved to hemidystonia or generalized dystonia with rapid deterioration in cognitive function in these patients. A few CJD cases with an atypical history of corticobasal degeneration, such as the alien hand phenomenon, apraxia and parkinsonism have also been reported
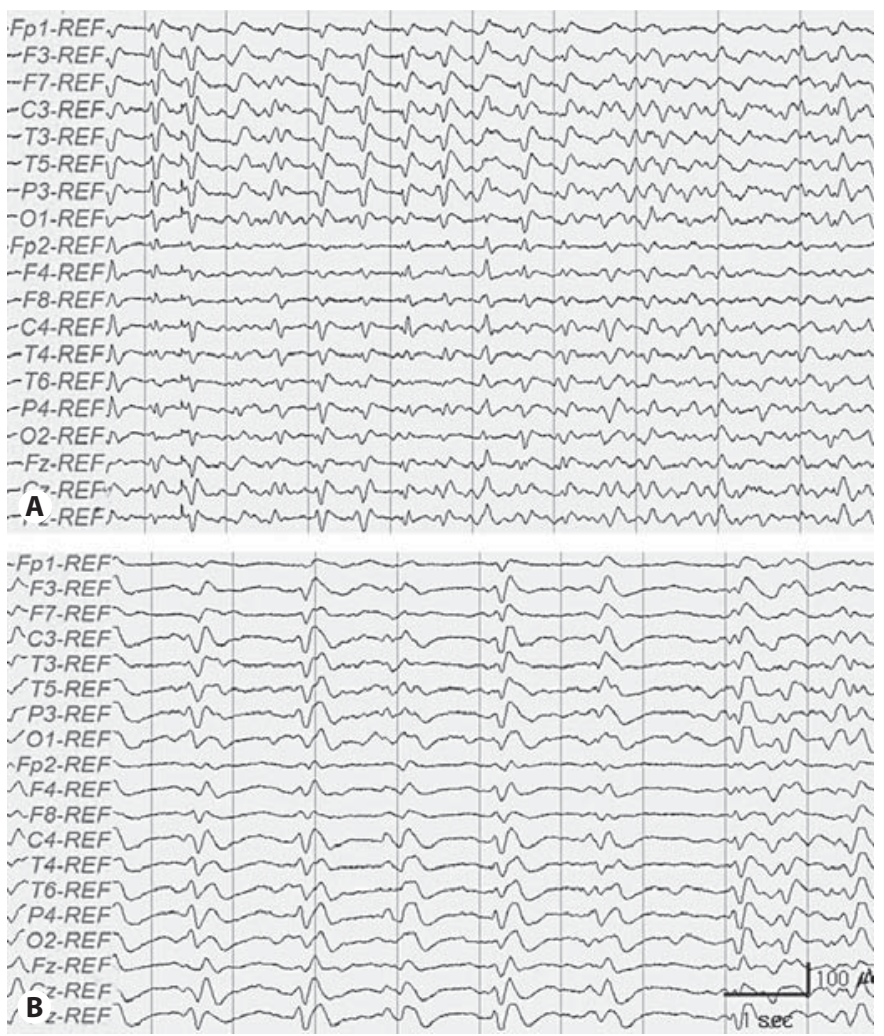

Fig. 2. EEG study. A EEG on the third hospital day shows findings typical of CJD: periodic asymmetrical sharp waves occurring at a frequency of $2 \mathrm{~Hz}$. B EEG on the tenth hospital day shows periodic generalized sharp waves occurring at a frequency of $0.5-$ $1 \mathrm{~Hz}$.

[10-12]. Our patient presented with unilateral dystonia, which evolved to generalized dystonia during the course of the disease; in addition, there were superimposed myoclonic jerks with rapid deterioration in cognitive and motor function. Parkinsonism and other clinical features of corticobasal degeneration were not observed.

In sporadic CJD, a bilateral symmetrical increased signal in the striatum on $\mathrm{T}_{2}$ weighted $\mathrm{MR}$ sequences is a distinctive finding; less commonly, asymmetrical involvement of the pallidum, thalamus and cerebral (especially occipital) cortex on $\mathrm{T}_{2}$ or proton density $\mathrm{MR}$ sequences is observed [13]. Some reports have shown typical MR findings of CJD $[2,6]$. However, prior reports have not shown the corresponding lesion associated with focal dystonia or involuntary movement on MR images.

DWI appears to be more sensitive than the standard MR sequences; it reveals high signal changes in the gray matter nuclei as well as the cerebral cortices $[13,14]$. In our case, the initial DWI revealed a corresponding striatal lesion with contralateral focal dystonia; the follow-up DWI also revealed bilateral striatal lesions, which correlated with generalized dystonia. These DWI findings suggest that the functional changes in the basal ganglia may be related to early dystonia in patients with CJD. However, abnormalities on DWI may not closely correlate with neuropathological findings (especially early in the course of CJD), suggesting a more functional, rather than anatomical, basis to the changes identified with DWI [15]. Therefore, we thought that most CJD patients, with unilateral DWI abnormalities, do not present with focal dystonia in early-stage CJD. In our report, the initial periodic sharp waves on EEG did not correspond to the DWI findings and the unilateral dystonia. Therefore, there was no correlation between the 
spreading dystonia and the periodic sharp waves.

This case illustrates that focal dystonia may occur in the early stage of CJD with functional changes noted in the basal ganglia.

\section{References}

1 Cooper SA, et al: Sporadic Creutzfeldt-Jakob disease with cerebellar ataxia at onset in the UK. J Neurol Neurosurg Psychiatry 2006;77: 1273-1275.

2 Donmez B, et al: Coexistence of movement disorders and epilepsia partialis continua as the initial signs in probable Creutzfeldt-Jakob disease. Mov Disord 2005;20:12201223 .

3 Hellmann MA, Melamed E: Focal dystonia as the presenting sign in Creutzfeldt-Jakob disease. Mov Disord 2002;17:1097-1098.
4 Josephs KA, Tsuboi Y, Dickson DW: Creutzfeldt-Jakob disease presenting as progressive supranuclear palsy. Eur J Neurol 2004;11:343-346.

5 Kirk A, Ang LC: Unilateral Creutzfeldt-Jakob disease presenting as rapidly progressive aphasia. Can J Neurol Sci 1994;21:350-352.

6 Maltete D, et al: Hemidystonia as initial manifestation of sporadic Creutzfeldt-Jakob disease. Eur J Neurol 2006;13:667-668.

7 Parry J, et al: Creutzfeldt-Jakob syndrome presenting as epilepsia partialis continua. J Clin Neurosci 2001;8:266-268.

8 Maltete D, et al: Movement disorders and Creutzfeldt-Jakob disease: a review. Parkinsonism Relat Disord 2006;12:65-71.

9 Marchiori PE, et al: Creutzfeldt-Jakob disease. A survey of 14 patients. Arq Neuropsiquiatr 1996;54:577-583.

10 Avanzino L, et al: Creutzfeldt-Jakob disease presenting as corticobasal degeneration: a neurophysiological study. Neurol Sci 2006; 27:118-121.
11 Kleiner-Fisman G, Bergeron C, Lang AE: Presentation of Creutzfeldt-Jakob disease as acute corticobasal degeneration syndrome. Mov Disord 2004;19:948-949.

12 Moreaud O, et al: Creutzfeldt-Jakob disease mimicking corticobasal degeneration clinical and MRI data of a case. J Neurol 2005; 252:1283-1284

13 Collins S, et al: Recent advances in the premortem diagnosis of Creutzfeldt-Jakob disease. J Clin Neurosci 2000;7:195-202.

14 Meissner B, et al: Sporadic Creutzfeldt-Jakob disease: magnetic resonance imaging and clinical findings. Neurology 2004;63:450456

15 Yee AS, et al: Diffusion-weighted MRI of right-hemispheredysfunctionin CreutzfeldtJakob disease. Neurology 1999;52:15141515. 